\title{
La géographie des phytotoponymes en Bretagne
}

\section{Samuel Perichon}

\section{(2) OpenEdition \\ Journals}

\section{Édition électronique}

URL : http://journals.openedition.org/abpo/1764

DOI : $10.4000 / a b p o .1764$

ISBN : 978-2-7535-1518-5

ISSN : 2108-6443

\section{Éditeur}

Presses universitaires de Rennes

Édition imprimée

Date de publication : 10 juillet 2010

Pagination : 9-24

ISBN : 978-2-7535-1214-6

ISSN : 0399-0826

\section{Référence électronique}

Samuel Perichon, «La géographie des phytotoponymes en Bretagne », Annales de Bretagne et des Pays de l'Ouest [En ligne], 117-2 | 2010, mis en ligne le 10 juillet 2012, consulté le 19 avril 2019. URL : http:// journals.openedition.org/abpo/1764; DOI : 10.4000/abpo.1764 


\title{
La géographie des phytotoponymes en Bretagne
}

\author{
Samuel PERICHON ${ }^{1}$ \\ Chercheur-associé, \\ ESO - université Rennes 2 Haute-Bretagne
}

La toponymie en tant qu'expression directe de la relation entre les lieux et leurs habitants successifs ${ }^{2}$ participe à la construction des territoires et à l'affirmation d'une identité régionale. La désignation des lieux de vie reproduit la manière dont chacun se positionne par rapport à la matérialité qui l'entoure; elle montre une inégale sensibilité au paysage et réveille des particularismes géographiques qui semblent liés à des motifs identitaires naturels ou construits. C'est sans doute pourquoi les modes d'appropriation de l'espace et, corrélativement, les stratégies de désignation, qui structurent le territoire et le transforment, varient grandement selon les époques et les régions.

Au temps du paganisme, la célébration des règnes minéral et végétal a contribué à l'émergence d'un paysage toponymique particulier. En effet, l'imaginaire suscité par les pouvoirs attribués à certains rochers, à certaines sources, à certaines plantes ${ }^{3}$, a inspiré de nombreux lieux-dits. Au-delà de la désignation, c'est-à-dire le fait de nommer un point dans l'espace, le toponyme fige le temps, d'où la survivance, très localement, d'anciennes croyances jusqu'à notre époque. À la faveur des grands défrichements accomplis à la fin du Moyen Âge, une nouvelle forme d'appropriation de l'espace apparaît ${ }^{4}$. Les dénominations louent désormais le courage et la détermination de leurs initiateurs ${ }^{5}$. Dans une moindre mesure, elles coïn-

1. L'auteur remercie Hervé GWEgEN (Responsable du service patrimoine, Office de la langue Bretonne, Carhaix), Marc CochARD (anc.), Bertrand TANGUY (Université de Bretagne Occidentale, Brest) et Pierre-Henri BILly (Institut d'Etudes Méridionales, Toulouse) pour leur relecture et leurs précieux conseils.

2. Cherpillod, André, Dictionnaire étymologique des noms géographiques, Masson, 1986, 527 p.

3. SÉBILLOT, Paul, La Flore, Imago, 1985 (réed. 1886), 215 p.

4. Deshayes, Albert, Dictionnaire des noms de lieux bretons, Chasse-Marée/ArMen, 2000, 256 p.

5. Durand, Jean-Yves, "Toponymie de la commune de Pléneuf ", Annales de Bretagne, 1960, p. 401-409. 
cident avec l'installation d'activités de production. À cet égard, ne pourrions-nous pas parler d'une laïcisation des toponymes? L'hypothèse est séduisante, même si l'engouement pour la production de biens de consommation, d'une façon générique, ne limite pas l'usage des hagiotoponymes. La construction d'ermitages et de chapelles ou, chose plus rare, la christianisation de petits oratoires champêtres ${ }^{6}$ l'attestent.

Au même titre que la topographie, l'hydrographie ou l'édification de lieux de culte, les plantes ont été une source d'inspiration non négligeable dans la construction du territoire. En Bretagne, plus de 4000 phytotoponymes ont été relevés, ce qui représente près d'un lieu-dit sur six ${ }^{7}$. Il s'agit majoritairement de noms désignant des bosquets, des bois, parfois des forêts (Annexes, tabl. 1), les populations leur ayant souvent adjoint des suffixes. Dans les pays de Rennes et de Loudéac, dans le Porhoët, le Poudouvre ou le Mené ${ }^{8}$, le recours à un patronyme est plus fréquent. Le choix reflète sans doute une volonté plus affirmée qu'ailleurs de marquer " son " territoire, le phytotoponyme intervenant comme un faire-valoir. Dans le Léon, le Trégor, le Poher ou le Haut-Vannetais, les noms de lieu intègrent davantage les boisements dans leur environnement naturel : sommet rocheux, motte, dépression, marais, cours d'eau, etc. L'examen des toponymes bretons d'arbres, d'arbustes et d'arbrisseaux, objet du présent article, précisera ces rapports différenciés aux territoires boisés ${ }^{9}$, il lèvera aussi le voile sur les préférences des populations et l'intensité du lien qui les unit aux végétaux.

Si la toponymie est une construction sociale, il n'en demeure pas moins qu'elle révèle très souvent l'aspect du lieu qu'elle désigne ${ }^{10}$, ce qui justifierait la place secondaire des plantes ligneuses dans le Finistère, un département sous influence maritime. Avec l'hypothèse d'un déterminisme physique dans le champ toponymique, c'est donc l'histoire du paysage végétal qui est proposée à la lecture ${ }^{11}$.

Pour faciliter le traitement des données, nous avons choisi de ranger les plantes en fonction de leur hauteur. Dans chacune des trois catégories, les " arbres", les " arbustes " et les " arbrisseaux", seules les espèces les plus fréquentes ont été analysées selon une logique d'échelles emboîtées (aires linguistiques, départements et/ou pays), elles ont ensuite été cartographiées. Le choix a été difficile à arrêter car nombre de plantes ligneuses, nombre de plantes semi-ligneuses, offraient des pistes de recherche intéressantes à explorer.

6. DuvAL, Michel, Mythologie des arbres en Bretagne, Royer/mythothèque, 2000, 202 p.

7. Ce travail a été réalisé à partir de la $B D N Y M E @$ de l'Institut Géographique National.

8. Voir Carte des pays traditionnels bretons, en annexe.

9. TANGUY, Bernard, "La forêt bretonne au Moyen Âge : le témoignage des textes et des noms de lieux ", BSAF, t. 135, 2006, p. 239-263.

10. GALLOIS, Lucien, Régions naturelles et noms de pays. Étude sur la région parisienne, 1909 (rééd. 2008), éd. CTHS, 350 p.

11. Desbordes, Jean-Michel, VilLOUTREIX, Marcel, "La toponymie de l'arbre en Limousin ", dans : Motтeт, Jean (dir.), L'Arbre dans le paysage, Paris, Champ-Vallon, coll. " Pays/ Paysages ", 2002, p. 90-102. 


\section{L'aulne, le chêne, le hêtre et le châtaignier}

La plupart des espèces de plantes mentionnées dans la toponymie bretonne sont des arbres d'espèces locales ou installées de longue date dans la région (72,4\%). En général, les toponymes font également référence à un type de boisement : un bosquet, une haie, un verger. Les plantes les plus répandues, - elles représentent 30,5\% de notre échantillon -, sont l'aulne et le chêne (tabl. 2). Le hêtre (7,3 \%) et le bouleau (5,1 \%) viennent ensuite. Le saule $(4,1 \%)$ et le frêne $(3,7 \%)$ se disputent la cinquième place. Derrière, un premier groupe se forme autour du poirier, du pommier, de l'orme, du châtaignier et du pin (2,3 à $3 \%)$; un deuxième rassemble le tilleul, le tremble, l'if, le cerisier et le cormier ( 0,9 à $1,5 \%)$; un dernier se dessine autour du peuplier, du merisier, du prunier, du sapin et du noyer (0,2 à 0,5\%). Les lieux-dits désignant un charme, un marronnier, un thuya, un érable ou un mimosa sont très rares $(0,1 \%)$, même si ponctuellement certains comme le mimosa, peuvent être assez fréquents.

\section{«Aulnaie » ou « marais »..., une polysémie qui pose problème}

D'une manière un peu abrupte, nous dirions que l'aulne ou plutôt l'aulnaie porte deux noms dans la région : gwern et Launay. En breton, gwern signifie aussi " marais ". Albert Deshayes, auteur d'un dictionnaire des noms de lieu bretons ${ }^{12}$ prend parti pour l'arbre en soulignant que dans les actes anciens, le mot a très souvent été rendu par l'Aulnais ou Launay. Une position plus consensuelle définirait le terme comme désignant une " zone humide boisée ou bordée d'aulnes ", ce qui ne disqualifie pas le lien avec l'arbre ${ }^{13}$.

Logiquement gwern se localise surtout en Basse-Bretagne, sous-région bretonnante. Le mot produit de nombreuses variantes, dont les plus connues sont guern et vern. La première concerne le Morbihan (69,7 \%) et les Côtes-d'Armor (63,6 \%), la seconde le Finistère $(59,8 \%)^{14}$. À la différence de vern qui, lorsqu'il est doublé, est associé à une orientation géographique, guern, ou guer par amuïssement, met en scène une diversité de champs. Les suffixes suggèrent une construction ou une activité : un moulin dans Guern-er-Vilin (Berné, 56), un puits dans Guern-ar-Punso (Saint-

12. Cet ouvrage a servi pour l'identification des toponymes de plantes en breton. Notre examen a été complété par l'utilisation d'un autre ouvrage de référence lorsqu'on étudie la fréquence des toponymes en Bretagne : Le Parcellaire Breton. Toponymes élémentaires, Association pour l'application de l'informatique aux études celtiques (AIEC), Institut culturel de Bretagne, 1986. Cet ouvrage répertorie 1500000 lieux-dits identifiés par la Direction générale des impôts dans son Répertoire informatisé des voies et lieux-dits, encore appelé le "Fichier Rivoli » (1974).

13. Le MoING, Jean-Yves, Noms de lieux en Bretagne : plus de 1200 noms expliqués, éd. Bonneton, 2004.

14. Dans la suite de l'article, pour ne pas allonger inutilement le texte, les noms des départements ont été remplacés par leur numéro. 


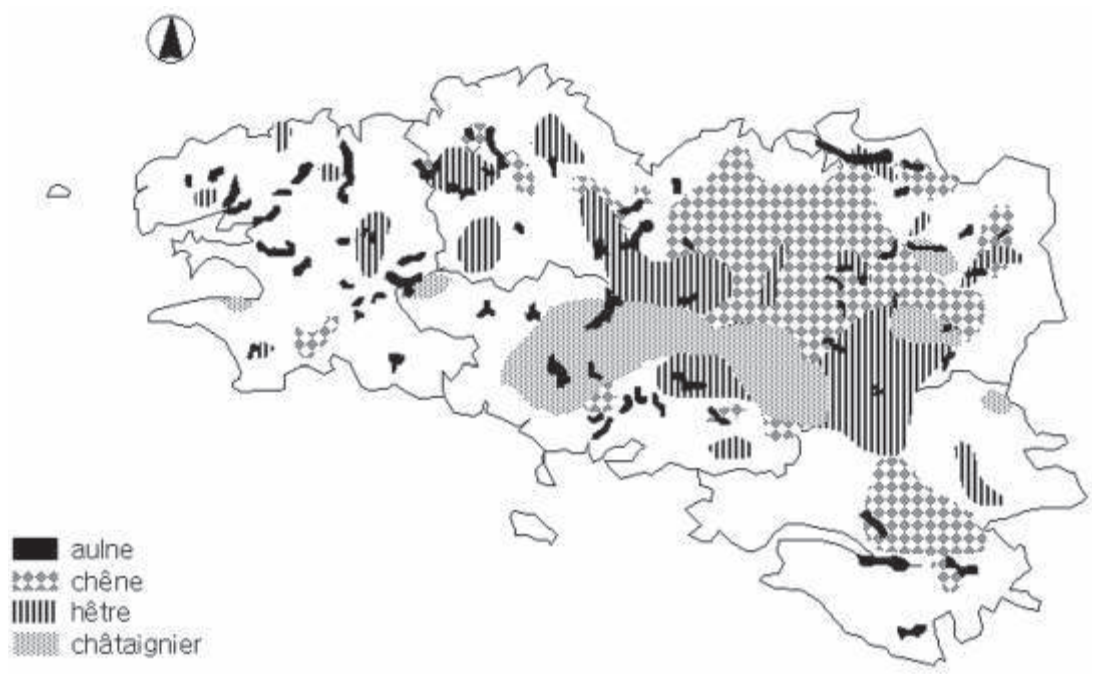

Agathon, 22), peut-être un ermitage dans Moustanguern ${ }^{15}$ (Plouyé, 29). Ils s'attardent vraisemblablement sur l'étendue du marais dans Le Guernhir (Langonnet, 56). Certains lieux-dits se rapportent à un patronyme : Guernar-Goff ${ }^{16}$ (Collorec, 29), Guernalain (Baud, La Chapelle-Neuve, 56), Guern Herve (Mousteru, 22) ou Gernisac (Taule, 29), plus rarement à un animal ${ }^{17}$ : Guern-an-Voualc'h (Gurunhuel, 22). En Haute-Bretagne, l'aulnaie se décline sous de multiples graphies : Aulnay bien sûr, mais aussi L'Aulnaie, L'Aunay, L'Aunaie, L'Aune, L'Aulnée, L'Aulneraie, etc. Contrairement aux habitants des autres pays haut-bretons, ceux du Dolois, du Clos Ratel et de Rennes ont utilisé des suffixes même s'il s'agit pour l'essentiel de patronymes.

L'hydrographie conditionne la localisation des lieux désignant l'aulne (carte 1). Ainsi, pouvons-nous suivre quelques méandres de l'Aulne, de l'Odet, de l'Elorn, de l'Aven, du Penzé (29), du Loc'h, l'aval du Blavet (56) et l'amont du Lié et de l'Oust (22). Excepté le pays de Rennes où les contours de la Seiche, du Meu et de la Vilaine apparaissent localement, le déterminisme hydrographique semble beaucoup moins évident à établir en HauteBretagne. En fait, l'aulne est surtout la plante de l'autre Bretagne, du Léon au pays Chtou, du pays Pourlet au Haut-Vannetais, l'arbre du Grand-Trégor aussi.

15. Les formes anciennes ne le confirment pas complètement, il peut s'agir d'une forme altérée de Mouz pour bourbier, ce qui renforcerait le sens de marais.

16. La probabilité pour que l'on ait affaire au patronyme Le Goff/Ar Gov est bien plus grande que le métier lui-même : forgeron. Ce patronyme serait en effet le quatrième nom le plus porté en Bretagne.

17. En l'occurrence, un merle. 


\section{Le chêne, l'arbre du Porhoët, du Mené, du Poudouvre, du Dolois, du Penthièvre, des Pays de Rennes et de Nantes}

Le chêne entre dans la composition de plus de 220 noms de lieux en Bretagne. Trois espèces sont signalées : le chêne pédonculé, le chêne rouvre et le chêne vert. Dans la région, le premier est, et de loin, le plus répandu : 89 \% de notre échantillon. En breton, le chêne pédonculé se dit derv ${ }^{18}$ dans Le Derff (Graces, 22), Le Boterf(f) (La Chapelle-Neuve, Naizin, 56), etc.; déro dans Coat-Déro (Combrit, 29), Clundéro (Roudouallec, 56), etc.; ou diry dans Le Diry (Locmaria-Plozané, 29), Ploudiry, 29), etc. Le chêne rouvre est indiqué dans Cosquer-Danouet et Tanouedou (Bourbriac, 22), Le Tanouet (Boquého, 22), etc. Le sens à donner à tann exige une grande prudence, car tan signifie aussi " feu ». Seuls deux, peut-être trois, toponymes en breton mentionnent un chêne vert : Calastren (Bangor, 56), Kerlastren (Plouguerneau, 29) et peut-être Kerastren (Plougasnou, 29). Les autres noms du chêne proviennent du latin ou du vieux-français. "Rouvre " procède de robur " roux ", il apparaît dans : Le Rouvre (Saint-Pierre-de-Plesguen, 35), (Le) Rouvray (Essé, Noyal-sur-Seiche, 35; Lanouée, 56; Joue-sur-Erdre, 44) ou La Rouvrais (Guichen, 35; Pluduno, 22). L'article défini valide une installation postérieure à l'an Mille ${ }^{19}$. Les noms issus du vieux français comme Chanais (Grandchamps-des-Fontaines, 44), Bocquenay (Questembert, 56), La Quennay (Guillac, 56), Le (petit) Chesnay (Plœuc-sur-Lié, Le Foeil, 22; Les Fougerets, 56)... sont surtout présents dans le pays de Nantes, dans le Poudouvre, le Penthièvre et le Mené où ils représentent jusqu'à $40 \%$ des toponymes de chêne. En Haute-Bretagne, la plante se retrouve plutôt sous les mots Chêne ou Chênaie, ce qui révèle un établissement récent même si l'arbre peut être très âgé. Si l'usage de mélioratifs n'est pas une nouveauté dans la toponymie, il est utile de mentionner que son usage à destination des phytoponymes se porte presque exclusivement sur le chêne. Les mélioratifs les plus banals étant : Beauchêne (Corps-Nuds, Bainde-Bretagne, Crevin, etc. : 35; Erbray, Saint-Marc-du-Désert, 44), Le grosChêne (Bazouges-la-Pérousse, Amanlis, Cornillé, 35; Casson, 44), Le grand Chêne (Vieillevigne, 44), etc.

Au regard de son importance dans le paysage végétal breton, la plante investit fort inégalement le territoire. À l'exception de quelques sites dans le pays de l'Aven, le Grand-Trégor, le Fanch ou le Haut-Vannetais, il faut admettre qu'en Basse-Bretagne, le chêne n'a guère inspiré de lieux (carte 1). Ailleurs la situation est différente bien que la diffusion du mot demeure imparfaite et son installation, tardive. Un très grand nombre de lieux-dits relatifs au chêne, 80 voire 90 , se localisent dans une large zone couvrant le Porhoët, le Mené, le Poudouvre, le Dolois, l'intérieur du Penthièvre et la moitié ouest du pays de Rennes. D'autres concentrations apparaissent;

18. Comme pour le patronyme Le Goff/Ar Gov, une confusion peut également se produire avec Derv, Le Deroff étant un nom de famille breton.

19. Desbordes, Jean-Michel, Villoutreix, Marcel, « La toponymie..., op. cit. 
la plus nette concerne le pays de Nantes avec plus d'un tiers des noms désignant un chêne vert.

\section{Le hêtre, l'arbre de la Bretagne intérieure : Poher, pays Fisel et de Loudéac, Porhoët}

Le hêtre intervient dans 140 noms environ. La plupart d'entre eux dérivent du latin fagus. D'un point de vue étymologique, hêtre provient du germanique heister, lequel serait à rapprocher d'un mode de gestion particulier de l'arbre ${ }^{20}$. Aussi est-il peu probable de le rencontrer sous cette forme avant le $\mathrm{XIII}^{\mathrm{e}}$ siècle. Orthographié haistre en ancien-français, le mot peine à se détacher de son sens initial, un régime en taillis. Par conséquent, la plante a longtemps été appelée fou, fouteau ou fousteau. Selon les époques et les pays, ces noms ont évolué, occasionnant une quantité impressionnante de variantes. Elles se sont d'abord construites à partir de fay, faux et feu. Citons : Fay-de-Bretagne (44), (Le) Fayet (Andouillé-Neuville, Ercée-enLamée, La Couyère, 35), Fayel(le) (Bourgbarré, Châteaubourg, La Couyère, 35), Le Faux (Guilliers, 56; Trévé, 22; Sixt-sur-Aff, 35), Le Feu (Soudan, 44), etc. "Fou " a fixé : Le Foue (Bain, 44), (Le) Biffou(e) (Loguivy-Plougas, 22; Guignen, 35), La (haute) Folie (Laurenan, Quessoy, Le Cambout, 22; Antrain, Gael, 35; Bourgneuf-en-Retz, 44), etc. Les noms en breton indiquant un ou plusieurs hêtres (fao, sing ${ }^{21}$, faven, plur.) ou une hêtraie (faouët) sont tout aussi abondants et diversement orthographiés. L'arbre est imagé dans : Le Fao (Huelgoat, 29), Kervao (Huelgot, Locmaria-Plouzané, Brest, etc. : 29), Ty-Faou (Plogonnec, 29), Run-Fao (Plougonver, 22), Fao-David, -Youen, -Glaz (Ploneour-Lanvern, 29), Penfao 22 (Saint-Thégonnec, 29) parfois francisé dans Painfaut (Saint-Vincent-sur-Oust, 56); dans Pulfaon (Le Faou, 29), Bodefa (Roudouallec, 56), etc. La plupart des noms sont précédés d'une indication géographique (kêr, lieu habité; ty, habitation; run, colline; pen, bout, extrémité; poul, étendue d'eau); d'un patronyme (David, Youen) ou bien l'aspect de la plante est précisé : glaz, verdoyant; bod, buissonnant. Visualiser une hêtraie est, semble-t-il, une chose plus aisée ainsi qu'en témoigne l'absence de préfixes : Le Faouet (La Chapelle-Neuve, Corlay, Le Faouet, 22; Plumergat, Plumelec, Le Faouet, 56). La formation végétale est également désignée sous le nom de " bois de hêtres " comme l'attestent: Folgoat (Pommerit-le-Vicomte, 22), Coueffaut (Peaule, 56) ou Fanhouët (Tréal, 56). Dans le Trégor, le Poher et le pays Fisel, les lieux-dits se répartis-

20. Domont, Philippe, Montelle, Édith, Histoire d'arbres, des sciences aux contes, Paris, Delachaux et Nestlé/Office National des Forêts, 2003, 220 p.

21. Dans la toponymie, le singulatif peut également désigner un boisement de la même essence; cf Divi Kervella, Petit Guide des Noms de Lieux Bretons, Spézet, éd. Coop Breizh, 2007, 120 p.

22. " Le toponyme peut être ambigu. En effet, il est connu que Penn peut provoquer la mutation ancienne du p- et f- à l'initiale du mot qui suit (Penn + Prad = Penfrad). Il se trouve que certains Penfao actuels recouvrent Penn + Paou qui revêt plusieurs sens, dont celui de "pays", circonscription ancienne, et qui apparaît dans certains noms de pays bretons, tels que Poher, Poudouvre, Porhoët, etc. " selon Hervé GwEGEN (OLB-Carhaix). 
sent autour de noyaux de taille modeste, de l'ordre de 10 à 15 km (carte 1), tandis qu'ils recouvrent, plus à l'est, une vaste zone comprenant le Porhoët, les pays de Loudéac et de Redon, et la moitié nord du Vannetais gallo. Cette distinction s'explique probablement par la nature de l'occupation du sol : les boisements bas-bretons sont en général morcelés et éparpillés alors qu'en Haute-Bretagne, la plante couvre de vastes massifs forestiers (forêts de Paimpont, de Loudéac, de Lanouée, de la Hardouinais ou de Lorge).

\section{Le châtaignier, l'arbre de la Bretagne méridionale : Bas-Vannetais, pays de Baud et de Pontivy}

Comme l'aire endémique du châtaignier couvre une région éloignée de la Bretagne, cela participe de sa place secondaire dans la toponymie : seulement 50 lieux-dits ont été recensés. Tous les noms du châtaignier dérivent du latin castanea. En breton, l'arbre se dit kistin avec comme dérivé Kistinid. Le mot est présent sous sa forme initiale dans Botquistin (Moustoir-Ac, 56), Quistinit, 56), Quistinic (Moustoir-Ac, Quistinic, Gourin, 56), Kerguistin (Saint-Gen, 22; Noyal-Pontivy, 56), etc., et sous des variantes dans Penquesten (Inzizac-Lochrist, Langonnet, 56), Bod-Kesten (Camors, 29), Kerguesten (Pouldergat, Douarnenez, 29), etc. En Haute-Bretagne, le châtaignier se retrouve dans Chany (Tremblay, 35), Chasné-sur-Illet (35) ou (Le) Chatenay (Cornillé, Paimpont, Corps-Nuds, Orgères, 35). La distribution des Châtaignier(s) et Châtaigneraie, c'est-à-dire des noms actuels, concerne principalement les pays de Rennes et de Redon. Les conditions du milieu, notamment l'ensoleillement, ont contribué à isoler une localisation préférentielle. L'acclimatation de la plante a été plus rapide dans la partie méridionale de la région (carte 1). Ainsi le Bas-Vannetais, les pays de Baud et de Pontivy, le tiers nord du Vannetais gallo et l'ouest du Porhoët regroupent près de $50 \%$ des toponymes désignant des châtaigniers. Leur distribution forme un arc de cercle d'une vingtaine de kilomètres de diamètre entre Plouhinec à l'ouest et Cournon à l'est, avec de fortes densités autour de Noyal-Pontivy, Inzinzac-Lochrist et Moustoir-Ac. Ponctuellement, l'intérêt des populations pour la plante se vérifie aussi dans les pays Penn Sardin et Chtou, et au nord de Rennes.

Avant même nos investigations, il ne faisait aucun doute, au regard de son importance dans la culture bretonne, que le chêne serait largement consacré. Il en allait bien autrement de l'aulne, cet arbre effacé, presque méconnu. Pourquoi lui avoir accordé une telle importance dans la toponymie? Il est difficile d'y répondre, même si la cartographie nous fournit des éléments de réponse. La plante a probablement été retenue en raison de son omniprésence en bordure de cours d'eau et dans les zones humides, ce qui nous ramène à la polémique sur le sens à donner à aulnay et gwern... Il est clair que dans ce cas précis, le double sens biaise les statistiques. De plus, sous forme lénifiée -vern, dans les noms composés comme Kervern ou Lanvern, il peut peut-être s'agir de bern " monticule ». À travers les arbres 
étudiés, l'étude a permis d'identifier deux constantes dans la désignation des lieux de sociabilité. La première relève du déterminisme physique, la seconde de l'intensité du lien qui rattache l'homme à la plante, l'une et l'autre intervenant à des degrés variables selon les espèces et les particularismes linguistiques.

\section{L'aubépine, le buis, le houx et le sureau}

Hors du Porhoët, du Léon et du pays de Plougastel, les noms d'arbustes sont très peu employés pour désigner des lieux en Bretagne. Moins d'un phytotoponyme sur six indique un arbuste : dans la plupart des cas il concerne l'aubépine (ou d'autres plantes à épines) et le buis (tabl. 3).

\section{L'aubépine et autres plantes à épines : Léon, Poher, Haut-Vannetais et Coglais}

L'influence des plantes à épines est bien supérieure au chiffre avancé dans le tableau annexé, car ce dernier ne prend pas toujours en compte les boisements linéaires qu'elles composent ${ }^{23}$. En Basse-Bretagne, trois noms se rapportent à des épineux dont l'aubépine : drein, spern et draeneg. Le premier mot forme : Cleudrain (Plésidy, Tréglamus, 22), Kerdrain (Saint-Caradec, Kerpert, 22), Quilliodren (Le Trevoux, 29), Kerdréan (Bono, Treffléan, Caudan, etc. : 56) ou Landrin (Plumelec, 56). En général, kêr précède spern ou bien il évoque un bois dans Coat Spern (Tourc'h, 29), une lande dans Lan Bot Spern (Locmaria-Grand-Champ, 56), l'aspect buissonnant dans Botspern (Bignan, 56). Draeneg intervient dans une quinzaine de noms parmi lesquels (Le) Drennec (Clohars-Fouesnant, Tourch, Sizun, etc. : 29), Le Drenidan (Radenac, 56) ou Coatrennec (Glomel, 22). En Haute-Bretagne, les dénominations sont Epinay et Epine. La première, la plus ancienne, fixe seize toponymes dont la plupart se localisent dans le Coglais. La seconde, plus fréquente, se pose sous une forme composée. L'information retient une activité humaine dans Port l'épine (Trelevern, 22) ou Bourg-L'épine (Louvigné-du-Désert, 35), elle précise une espèce dans Les épines blanches (Langueux, 22) ou L'épine rouge (La Bouexière, 35), une localisation dans l'épine des haies (Guéméné-Penfao, 44) ou peut-être un mode de gestion dans Le Plessis-Lépine (Cuguen, 35). Plusieurs variantes ont été identifiées : Les Epinettes (Domalain, Ercé-en-Lamée, 35; Saint-Gildasdes-Bois, Coueron, 44), Les Epiniaux (Pacé, 35), Epineu (Bourg-les-Comptes, 35), Épiniac (35) ou Fleurigné (35).

Plus d'un toponyme sur trois se situe dans le Léon, dans les pays Bidar, Glazic et Bigouden (carte 2). Dans la majorité des cas, les noms sont localisés en bordure de mer. Dans le département du Finistère, l'arbuste occupe la deuxième place au classement des phytotoponymes les plus fréquents, chose insolite à l'échelle de la région. Ailleurs en Bretagne, les noms de

23. AnToInE, Annie, Le paysage de l'historien, Rennes, PUR, 2002, 340 p. 
lieu se rapportant à l'aubépine sont surtout installés à l'intérieur des terres, comme dans le Coglais, les pays de Nantes et de Loudéac, au sud de Rennes, le Dolois ou le Haut-Vannetais.

\section{Le buis et les archéologues :}

Porhoët, pays de l'Aven, Dardoup et Bas-Trégor

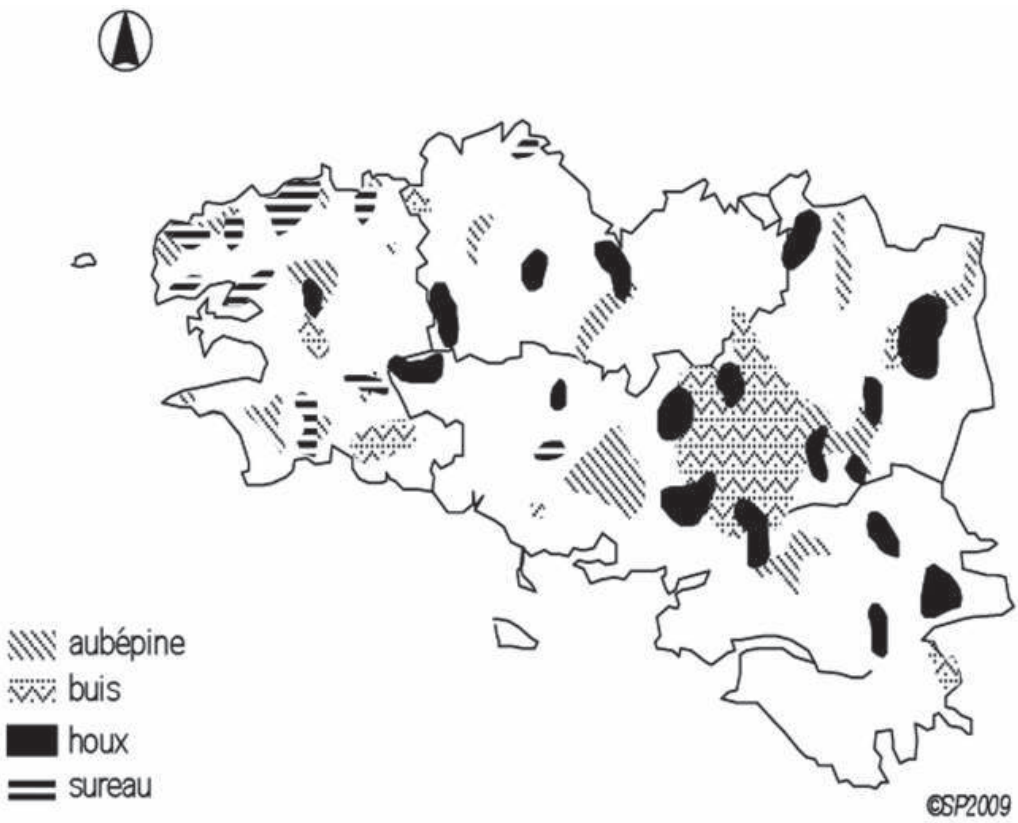

Le latin buxus buis, façonne une centaine de noms, les plus illustres étant La Boissière (Trégunc, Concarneau, Bannalec, etc., 29; Nivillac, Elven, Quelnec, etc., 56; Merléac, Yvignac, Tréglamus, etc., 22; Combourg, Vieux-Vy-sur-Couesnon, Rénac, 35; Soudan, Vigneux-de-Bretagne, 44), La Bouexière (Parigné, La Bouexière, Goven, etc., 35) et La Bouessière (SaintOnen-la-Chapelle, Bovel, Campel, etc., 35). Ces formes francisées sont présentes dans l'ensemble de la région ${ }^{24}$. En breton, le buis se dit beuz, la boissière, beuzid/ar veuzid. Nous les retrouvons dans : (Le) Buzit (Tréogon, Mellac, Lothey, etc., 29), Le/Ar Veuzit (Lanvollon, 22; Tréduder, Plestin-lesGrèves, 29), Buzidiou (Arzano, 29), Buzudel (Plounevez-du-Faou, 29) ou le

24. " En fait, on trouve les formes francisées La boissière et ses autres variantes francisées dans certaines sources... françaises. En revanche, dans la partie bretonnante, la plupart de ces toponymes sont employés uniquement dans leurs formes bretonnantes du lieu, soit ar veuzid, ar vujid, bezidell, etc. On ne peut donc pas parler de généralisation des formes françaises sans tenir compte des pratiques locales réelles ", selon Hervé GWEgen (OLB-Carhaix). 
Bezy (Caro, 56). Une localisation géographique peut être ajoutée ${ }^{25}$ pour : Beuzit-Braz (Trégonneau, 29), Braz-Vezit ${ }^{26}$ (Brech, 56), Le grand Veizit (Baden, 56). Dans la région, le buis a longtemps été une plante sacrée ${ }^{27}$. En cela, la distribution des toponymes anciens pourrait dévoiler des emplacements de culte. Certes, leur présence n'est pas toujours le gage de découvertes archéologiques majeures, mais les correspondances sont parfois troublantes ${ }^{28}$.

La carte $\left(n^{\circ} 2\right)$ met en évidence une zone circulaire d'une quarantaine de kilomètres centrée sur le pays Porhoët, où près de cinquante toponymes de buis ont été relevés. L'emplacement est emblématique dans le sens où il coïncide à peu près avec les limites de l'antique forêt de Brocéliande, lieu hiératique des traditions celtes. Hors de ce cadre géographique mais toujours en liaison avec les traditions et les croyances anciennes, il est saisissant de constater qu'une part non négligeable des noms investit des estuaires, pays de l'Aven et Bas-Trégor.

\section{Le houx, arbuste des sous-bois et lisières des forêts de Haute-Bretagne : Clos Ratel, pays de Rennes, de Redon et d'Ancenis}

Le houx inspire soixante et onze lieux dont près de cinquante indiquent une houssaie. En breton, l'arbuste est nommé kelenn. Le mot porte habituellement un suffixe -eg, ce qui est une configuration exceptionnelle pour un boisement : Quelennec, Quelneuc, Quenelec et Quenelet. La remarque vaut pour les termes en français. Si La Houssais (Landavran, Muel, Domloup, etc., 35; Saint-Vincent-des-Landes, Sévérac, Rezé, 44), La Houssaye (Iffendic, Redon, 35; Quessoy, Plédran, 22; Saint-Thuriau, Pontivy, 56), Le Houssay (Livre-sur-Changeon, 35; Sucé-sur-Erdre, 44) révèlent la présence passée de la plante aux lieux cités, d'autres comme Les Houssines (Ercé-en-Lamée, 35), La Houssinais (Guichen, 35), peut-être La Housselais (Saint-Aubin-duCormier, 35), informent sur une ancienne activité de production de balais de houx.

Trois toponymes sur cinq se localisent en Ille-et-Vilaine ou dans le Morbihan. L'arbuste s'établit plutôt à l'intérieur des terres, souvent à proximité de petits massifs forestiers se situant non loin d'une ligne allant du

25. En réalité, l'adjectif bras, grand, ne se rapporte pas au nom commun beuz-beuzid/ar veuzit, mais à la taille du village ainsi dénommé. Pour preuve, on retrouve fréquemment un Beuzit-vihan ou Ar veuzit vihan (bihan, petit) à proximité du grand. Comme bihan et bras, la toponymie bretonne est riche de nevez et kozh (nouveau et ancien) qui se rapportent, eux, à l'époque de la création du village et non pas au qualificatif du nom. D'après Hervé GwEngen (OLB-Carhaix).

26. Configuration surprenante s'agissant de l'ordre des termes.

27. Duval, Michel, Mythologie des arbres..., op. cit., p. 109.

28. GHestem, Axel, " La végétation ligneuse révélatrice des vestiges archéologiques ", in MotTet, Jean (dir.), L'Arbre dans le paysage, Paris, Champ-Vallon, coll. " Pays/Paysages ", 2002, p. 78-89. 
pays Fisel à l'ouest, au pays d'Ancenis au sud-est (carte 2). Le long de cet axe, gravite une quinzaine de poches de superficie modeste. Signalons une densité assez forte aux abords des forêts de Liffré (nord-est de Rennes), de Coëtquen (Clos Ratel) et du Cranou (pays Rouzig).

\section{Le sureau, l'arbuste du Finistère : Léon, pays de Plougastel et de l'Aven}

Le sureau s'inscrit pleinement dans la dualité Haute/Basse-Bretagne. Cela paraît difficile à admettre mais l'arbuste se manifeste presque exclusivement sous un vocable breton; en langue gallèse, le sureau se dit su d'où la Suais. De nombreuses graphies ont été identifiées : dans certaines (-scao, -sco, -scot, -scoff) il s'agit du collectif skav, dans d'autres (caven, scaven) du singulatif skavenn, et dans skavennoù de plusieurs sureaux mais considérés un par un. La terminaison -id, que l'on retrouve dans squivit, désigne aussi un lieu planté de sureau. Les noms sont très souvent précédés par kêr: kerscao (Quimper, Trégourez, Beuzec-Cap-Sizun, etc., 29), Kerscoff (Scaër, Morlaix, 29), Kersco (Peumerit, 29), Kerscot (lle-aux-Moines, 56). Parfois comme dans Poulscavennou (Cléder, 29) ou Poulscaven (Lothey, 29), les dénominations renseignent sur les préférences édaphiques de l'arbuste : Poul, flaque d'eau, mare. La quasi-totalité des toponymes sont localisés dans le Finistère, avec une très forte densité dans le Léon (PlounevezLochrist), et dans une moindre mesure dans les pays de Plougastel et de l'Avent.

\section{Le noisetier, le genêt et la bruyère}

Parmi les plantes étudiées, les arbrisseaux sont très peu représentés dans le paysage toponymique, tout au plus constituent-ils $10 \%$ de notre échantillon. Le noisetier et le genêt forment l'essentiel des lieux-dits (7,3\%), la bruyère n'occupant qu'une place marginale (tabl. 4).

\section{Le noisetier, l'arbrisseau du pays Fanch, des terres intérieures du Trégor et du pays de Rennes}

Le noisetier entre dans la composition de quatre-vingt-un noms. Le mot tel que nous l'employons aujourd'hui est d'usage récent, il remonterait au début du $\mathrm{XVI}^{\mathrm{e}}$ siècle ${ }^{29}$. Longtemps "noisetier" a été précédé par l'ancien français coudre ou coudrier, qui n'est autre que la déformation du latin corylus. En Haute-Bretagne, La Noisetterie (Montbert : 44) est l'unique exemple de noms procédant de la dénomination actuelle de l'arbrisseau. Neuf fois sur dix, les noms anciens sont posés seuls et ils représentent un lieu abondamment fourni. Jusqu'au XIX ${ }^{\mathrm{e}}$ siècle, La Coudray et Les Coudreaux, c'est-àdire les noms plus anciens, sont privilégiés avec de menues corrections :

29. BRosse, Jacques, Larousse des arbres et des arbustes, Paris, Larousse, 2001, 576 p. 
La Coudray devient La Coudrais puis La Coudraie. En Basse-Bretagne, les plantations de noisetiers sont signalées par deux noms : koll et kelwez, le premier nom étant le plus courant, 20 des 25 toponymes. À la différence des lieux-dits issus du français, ceux d'origine bretonne sont, une nouvelle fois, composés. Dans la majorité des cas, ils sont associés à kêr. Les autres signalent : un cours d'eau dans Gollot la rivière (Carnoët, 22), un marais dans Guergolvez (Plouaret, 22), une dépression humide dans Toul an golet (Plésidy, 22) ou une prairie dans Prat collet ${ }^{30}$ (Pleudaiel, 22). La proximité à un village est évoquée dans Gollot Braz (Louargat, 22) et Collet Meur (Plédernec, 22).

C'est surtout dans le pays Fanch, le Petit-Trégor et l'intérieur du Penthièvre que l'arbrisseau produit un nombre significatif de lieux-dits (carte 3) : il va jusqu'à se placer dans le carré de tête des phytotoponymes costarmoricains (tabl. 4). Ailleurs, la distribution des noms s'organise autour de poches de quelques kilomètres de diamètre situées dans les pays de Rennes, de Redon, d'Ancenis et dans le Coglais.

\section{Le genêt des landes de bord de mer: pays Pagan, Crozon, Penn Sardin et Penthièvre}

Une soixantaine de lieux évoque le genêt. Une nouvelle fois, les configurations bretonnes diffèrent de celles de langue romane. Huit fois sur dix, l'arbrisseau, appelé balan, est associé avec ker-, Tre- " hameau " ou Lann- lieu consacré ou " lande, terre inculte où pousse l'ajonc ". Nous les retrouvons dans : Kervalan (Saint-Péver, 22; La Forest-Landerneau, BourgBlanc, 29; Melrand, 56) ou Kerbalanec (Lannion, 22; Beuzec-Cap-Sizun, Pencran, 29). En français, le nom est posé seul : La Genière (Hénon, 22), La Ginière (Martigné-Ferchaud, 35), Le Genetay (Messac, 35) ou Les Genêts (Trémaouezan, 29; Saint-Médard-sur-Ille, 35). Les landes littorales du Léon, de Penthièvre, du Grand-Trégor, des pays de Plougastel, de Crozon et de Penn Sardin regroupent à elles seules près de deux tiers des toponymes de genêt en Bretagne. La plante est également mentionnée dans les landes des pays de Retz et de l'Aven, et dans le Guerchais (carte 3).

\section{La bruyère des landes du Poher, du Guerchais et des pays de Rennes et de Baud}

La bruyère fixe trente-six noms, dont dix proviennent du breton brug. La localisation du terme breton est inattendue car il investit indifféremment les deux aires linguistiques : Bruz (35), Bruc-sur-Aff (35), Brutz (Soulvache, La Chapelle-Glain, 44) ou Bruguel (Derval, 44). Dans le Finistère et dans les Côtes-d'Armor, les noms sont composés : Menez Brug (Fouesnant, 29), Kerbruc (Scaër, La Feuillée, 29), Trébruc (Saint-Hernon, 29) ou Lanvruc ar bize (La Chapelle-Neuve, 22). En vieux-français, " bruyère " se disait

30. À moins que prat collet ne signifie praire perdue. 
" bruère " ou " brière ", mais quelle que soit leur ancienneté, les dénominations intéressent un même nombre de lieux lesquels sont surtout présents dans le pays de Rennes et dans le Guerchais (carte 3). En Basse-Bretagne, deux zones semblent particulièrement sensibles à la plante, le Poher et le pays de Baud.

L'examen des phytotoponymes devait nous permettre d'identifier le paysage végétal breton durant des périodes assez anciennes. Pour cela, il fallait se garder de tomber dans les pièges de cette science; l'origine d'un lieu-dit est une chose, dater son installation en est une autre... car nombre de noms issus de langues mortes ont en réalité été établis durant le haut Moyen Âge. D'ailleurs il est vraisemblable que la grande majorité des noms que nous avons traités date précisément de cette époque.

À la lumière des données recueillies, le paysage breton était façonné par une anthropisation assez ancienne : il était planté de bosquets de chêne et de hêtre, et fermé dans le Porhoët par une vaste forêt, la forêt de Paimpont. À travers les cultes païens qui sans doute y étaient célébrés, ce lieu a non seulement favorisé la célébration d'autres plantes dans la toponymie comme le buis ou le houx, mais il a surtout modifié le regard porté sur le végétal. La Bretagne se caractérisait également par l'étendue des terres de lande, ce motif paysager étant fixé d'emblée ou suggéré par un emploi métonymique.

Pour la plupart des plantes, le déterminisme physique joue un rôle décisif dans la diffusion et la répartition spatiale des toponymes. L'aulne par exemple s'installe préférentiellement dans les zones humides et en bordure des cours d'eau, d'où sa fréquence dans la région des monts d'Arrée ${ }^{31}$, et plus généralement sur l'ensemble du réseau hydrographique finistérien. L'hypothèse se vérifie aussi dans les pays du Léon et de Plougastel, pour un arbuste comme le sureau. Cette plante est d'ailleurs riche d'enseignements, car si elle appuie la logique déterministe, elle démontre dans le même temps que le choix porté sur telle ou telle plante n'est pas neutre. Ce choix répond en effet à des critères esthétiques, il renvoie à une dimension symbolique voire affective, ou plus prosaïquement il satisfait à des impératifs fonctionnels.

La construction des phytotoponymes, et cela indifféremment de leur densité sur le territoire breton, est marquée par cette perception différenciée du végétal, par des préférences qui sont toutefois susceptibles d'évoluer dans le temps et l'espace. L'usage très restreint d'un mélioratif apporte indiscutablement la preuve d'une affinité plus forte avec certaines plantes comme le chêne. Les aires linguistiques interviennent aussi dans la nature et l'intensité des relations avec les végétaux du paysage ordinaire. D'une manière un peu caricaturale, l'examen des phytotoponymes montre l'im-

31. La prédominance de l'aulne dans les statistiques ne semble pas fondé, car ce terme correspondant majoritairement au mot marais. 
portance de la géographie dans la lecture des territoires boisés en BasseBretagne, tandis que l'orientation dans la sous-région de langue romane repose davantage sur une lecture du foncier.

Malgré les recherches engagées, de nombreuses zones d'ombre subsistent, en particulier pour les noms de lieu d'origine bretonne. Un examen systématique de leurs formes historiques aurait indiscutablement mérité une attention particulière. De même, la portée des hagiotoponymes a été sous-estimée dans notre étude. Il aurait été également intéressant de mener des investigations complémentaires à partir d'autres sources d'informations que la $B D N Y M E @$. Cela aurait permis, mais c'est un autre travail, de confronter le paysage toponymique et sa réalité visible au moment où il s'est construit, chose qui, dans notre étude, n'a malheureusement pas été à la hauteur de nos espérances.

\section{Annexes}

\section{Carte 1 - Les « pays » bretons traditionnels}

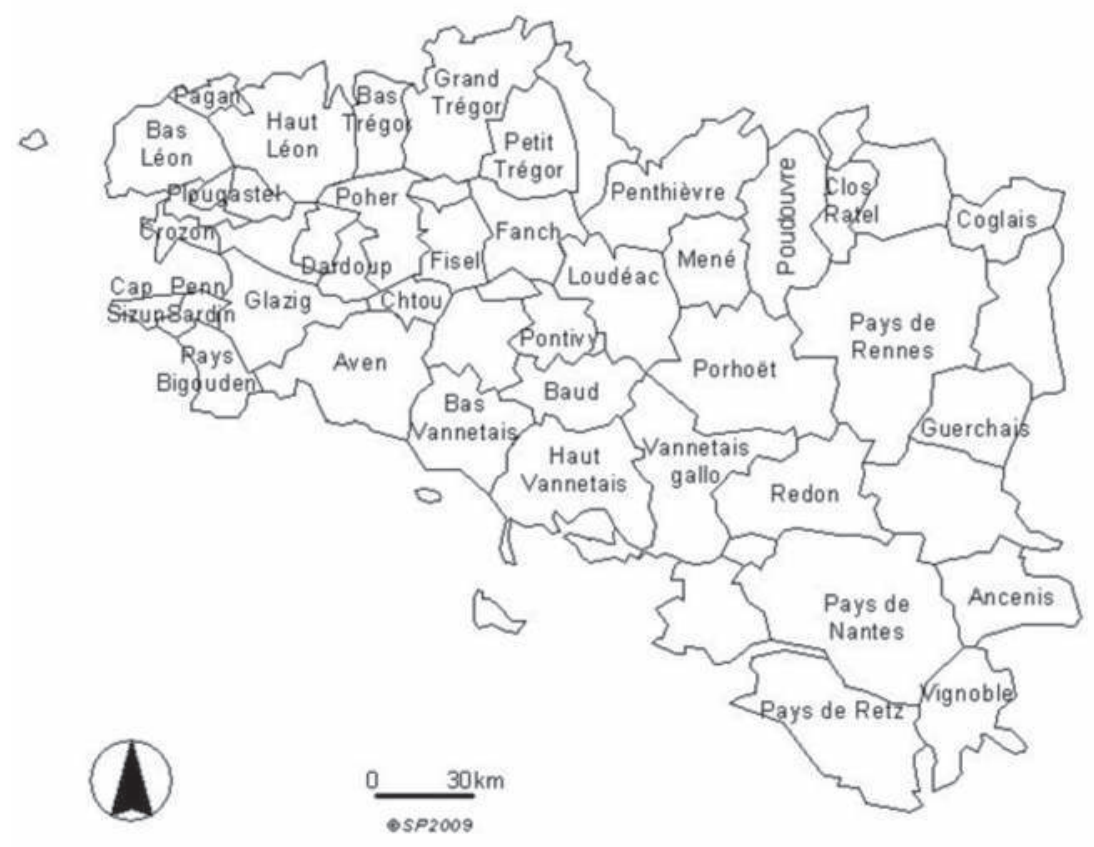


La géographie des phytotoponymes en Bretagne

Tableau 1 - Les plantes ligneuses dans la toponymie bretonne

\begin{tabular}{|c|c|c|c|c|c|c|c|}
\hline & & Finistère & Morbihan & $\begin{array}{l}\text { Côtes- } \\
\text { d'Armor }\end{array}$ & $\begin{array}{l}\text { Ille-et- } \\
\text { Vilaine }\end{array}$ & $\begin{array}{c}\text { Loire- } \\
\text { Atlantique }\end{array}$ & Bretagne \\
\hline \multirow[t]{2}{*}{$\begin{array}{l}\text { Forma- } \\
\text { tions } \\
\text { végétales }\end{array}$} & $\begin{array}{l}\text { bois, forêt } \\
\text { lande } \\
\text { haie } \\
\text { brous- } \\
\text { sailles } \\
\text { verger }\end{array}$ & $\begin{array}{c}72,8 \% \\
10,3 \% \\
6,7 \% \\
5,7 \% \\
4,5 \% /\end{array}$ & $\begin{array}{c}71,9 \% \\
12,0 \% \\
6,2 \% \\
7,0 \% \\
2,9 \%\end{array}$ & $\begin{array}{c}61,1 \% \\
16,7 \% \\
13,9 \% \\
5,3 \% \\
2,9 \%\end{array}$ & $\begin{array}{c}63,5 \% \\
14,3 \% \\
14,8 \% \\
2,5 \% \\
4,9 \%\end{array}$ & $\begin{array}{c}55,8 \% \\
16,3 \% \\
19,9 \% \\
4,2 \% \\
3,8 \%\end{array}$ & $\begin{array}{c}65,8 \% \\
13,7 \% \\
11,7 \% \\
4,9 \% \\
3,8 \%\end{array}$ \\
\hline & total & 10,1 & 10,4 & 10,5 & 11,0 & 10,6 & 10,5 \\
\hline \multirow[t]{2}{*}{$\begin{array}{l}\text { espèces } \\
\text { végétales }\end{array}$} & $\begin{array}{l}\text { arbres } \\
\text { arbustes } \\
\text { arbris- } \\
\text { seaux }\end{array}$ & $\begin{array}{c}61,7 \% \\
28,4 \% \\
9,9 \%\end{array}$ & $\begin{array}{c}74,3 \% \\
18,1 \% \\
7,6 \%\end{array}$ & $\begin{array}{l}75,2 \% \\
13,0 \% \\
11,8 \%\end{array}$ & $\begin{array}{c}75,3 \% \\
15,6 \% \\
9,1 \%\end{array}$ & $\begin{array}{l}75,9 \% \\
13,4 \% \\
10,7 \%\end{array}$ & $\begin{array}{c}72,4 \% \\
17,9 \% \\
9,7 \%\end{array}$ \\
\hline & total & 5,5 & 7,3 & 6,6 & 9,6 & 6,1 & 7,3 \\
\hline \multicolumn{2}{|c|}{$\begin{array}{l}\text { phytotoponymes / } \\
\text { total }\end{array}$} & 12,6 & 16,6 & 17,0 & 18,6 & 13,6 & 15,6 \\
\hline
\end{tabular}

Tableau 2 - Les arbres dans la toponymie

\begin{tabular}{|l|c|c|c|c|c|c|}
\hline & Finistère & Morbihan & $\begin{array}{c}\text { Côtes- } \\
\text { d'Armor }\end{array}$ & $\begin{array}{c}\text { Ille-et- } \\
\text { Vilaine }\end{array}$ & $\begin{array}{c}\text { Loire- } \\
\text { Atlantique }\end{array}$ & Bretagne \\
\hline aulne & $25,7 \%$ & $21,5 \%$ & $20,3 \%$ & $14,2 \%$ & $10,7 \%$ & $18,6 \%$ \\
chêne & $4,6 \%$ & $10,8 \%$ & $15,8 \%$ & $15,4 \%$ & $12,3 \%$ & $11,9 \%$ \\
hêtre & $6,4 \%$ & $8,1 \%$ & $9,1 \%$ & $6,3 \%$ & $6,9 \%$ & $7,3 \%$ \\
bouleau & $1,1 \%$ & $7,3 \%$ & $2,7 \%$ & $7,0 \%$ & $6,5 \%$ & $5,1 \%$ \\
saule & $3,5 \%$ & $4,2 \%$ & $4,8 \%$ & $3,8 \%$ & $4,6 \%$ & $4,1 \%$ \\
frêne & $3,2 \%$ & $2,4 \%$ & $3,6 \%$ & $4,4 \%$ & $5,4 \%$ & $3,7 \%$ \\
châtai- & $1,6 \%$ & $5,5 \%$ & $0,6 \%$ & $2,7 \%$ & $2,7 \%$ & $2,7 \%$ \\
gnier & $15,6 \%$ & $14,5 \%$ & $18,2 \%$ & $21,6 \%$ & $26,9 \%$ & $19,0 \%$ \\
autres & $61,7 \%$ & $74,3 \%$ & $75,1 \%$ & $75,4 \%$ & $76 \%$ & $72,4 \%$ \\
\hline \multicolumn{1}{|c|}{ total } & & & & & \\
\hline
\end{tabular}

Tableau 3 - Les arbustes dans la toponymie

\begin{tabular}{|l|c|c|c|c|c|c|}
\hline & Finistère & Morbihan & $\begin{array}{c}\text { Côtes- } \\
\text { d'Armor }\end{array}$ & $\begin{array}{c}\text { Ille-et- } \\
\text { Vilaine }\end{array}$ & $\begin{array}{c}\text { Loire- } \\
\text { Atlantique }\end{array}$ & Bretagne \\
\hline aubépine & $11 \%$ & $5,2 \%$ & $5,5 \%$ & $6,8 \%$ & $4,6 \%$ & $6,1 \%$ \\
buis & $5,4 \%$ & $6,0 \%$ & $4,2 \%$ & $4,4 \%$ & $4,6 \%$ & $5,6 \%$ \\
houx & $3,5 \%$ & $5,2 \%$ & $1,2 \%$ & $4,4 \%$ & $4,2 \%$ & $3,8 \%$ \\
sureau & $8,6 \%$ & $1,6 \%$ & $2,1 \%$ & $0 \%$ & $0 \%$ & $2,4 \%$ \\
autres & $0 \%$ & $0,3 \%$ & $0 \%$ & $0 \%$ & $0 \%$ & $0,1 \%$ \\
\hline \multicolumn{1}{|c|}{ total } & $28,5 \%$ & $18,3 \%$ & $13 \%$ & $15,6 \%$ & $13,4 \%$ & $18 \%$ \\
\hline
\end{tabular}


Tableau 4 - Les arbrisseaux dans la toponymie

\begin{tabular}{|l|c|c|c|c|c|c|}
\hline & Finistère & Morbihan & $\begin{array}{c}\text { Côtes- } \\
\text { d'Armor }\end{array}$ & $\begin{array}{c}\text { Ille-et- } \\
\text { Vilaine }\end{array}$ & $\begin{array}{c}\text { Loire- } \\
\text { Atlantique }\end{array}$ & Bretagne \\
\hline noisetier & $2,7 \%$ & $3,9 \%$ & $7,0 \%$ & $4,7 \%$ & $3,1 \%$ & $4,3 \%$ \\
genêt & $5,6 \%$ & $1,3 \%$ & $2,7 \%$ & $1,7 \%$ & $5,0 \%$ & $3,0 \%$ \\
bruyère & $1,3 \%$ & $1,6 \%$ & $1,8 \%$ & $2,5 \%$ & $2,3 \%$ & $1,9 \%$ \\
autres & $0,3 \%$ & $0,8 \%$ & $0,3 \%$ & $0,2 \%$ & $0,4 \%$ & $0,4 \%$ \\
\hline \multicolumn{1}{|c|}{ total } & $9,9 \%$ & $7,6 \%$ & $11,8 \%$ & $9,1 \%$ & $10,8 \%$ & $9,6 \%$ \\
\hline
\end{tabular}

\section{RÉSUMÉ}

L'étude des noms de lieux, de leur origine, de leurs rapports avec les langues parlées ou disparues nous livre de précieuses informations quant à l'évolution des relations entre les sociétés et leur environnement. Au même titre que la topographie ou l'installation des premières structures religieuses, les plantes ont été une source d'inspiration importante dans la construction sociale des territoires. Ces phytotoponymes lèvent à la fois le voile sur les préférences des populations habitantes et l'intensité de leur lien avec les plantes, mais surtout ils décrivent un milieu plus ou moins façonné par l'homme. Le présent article propose de dresser un inventaire des noms de lieux d'origine végétale en Bretagne et d'en étudier la distribution spatiale.

\section{ABSTRACT}

A strategy more or less purposeful to name the place of life is necessary to appropriate a territory. Thus the toponymy concretises a frame of mind specific to particular sociocultural contexts, it conveys the way which societies face up to an environment. Within this framework, the plants, like topography or hydrography were useful, and from time immemorial, to locate them. This paper proposes to draw up an inventory of the phytotoponyms in Britany and to study their spatial distribution. 